\title{
Frequency analysis of five short periodic pulsators
}

\author{
A. Liakos \& P. Niarchos \\ National and Kapodistrian University of Athens, Faculty of Physics, \\ Department of Astrophysics, Astronomy and Mechanics, \\ GR 157 84, Zografos, Athens, Hellas
}

\begin{abstract}
CCD observations of five newly discovered pulsating stars are analyzed using the Period04 software for a comprehensive investigation of their pulsation properties. The type of their variability is discussed, and the pulsation frequencies and Fourier spectra are presented.

Accepted: February 22, 2011

Individual Objects: TYC 1134-414-1, TYC 1270-926-1, TYC 1626-1303-1, TYC 3164-1517-1, TYC 4559-2536-1
\end{abstract}

\section{Introduction, Observations and data reduction}

TYC 1134-414-1 (=HIP108139 = HD 208238) was discovered as pulsating variable by Turner et al. (2008). They reported a dominant period of 0.048622 days and a spectral class of $A 3$, therefore the star was classified as $\delta$ Scuti type pulsator.

The light variability of the stars: TYC 1270-926-1 (=GSC 01270-00926), TYC 1626-1303-1 (=GSC 01626-01303), TYC 3164-1517-1 (=BD+43 $3593=$ GSC 03164-01517) and TYC 4559-2536-1 (=GSC 04559-02536) was reported by Liakos \& Niarchos (2011) as by-product in the field of view of other targets. TYC 3164-1517-1 is classified as F0 type star according to the AGK3 Catalogue (Heckmann 1975).

Preliminary results of the analysis of their light variations showed that lowamplitude and relatively quick periodic pulsations ( $45 \mathrm{~min}-3 \mathrm{hrs}$ ) occur. Such a variability corresponds to typical pulsational behaviour usually met in $\delta$ Scuti and $\beta$ Cephei stars, which are of A-F and B spectral type, respectively. Therefore, after the discovery of these variables, we performed follow up observations 
using appropriate binning modes and exposure times in order to obtain a comprehensive study of their variations. Given that these kinds of stars are hot in general, their largest pulsation amplitude is expected to occur in B-band, thus, the $B$ filter was selected for the observations. The time resolution along with the highest possible photometric signal-to-noise ratio $(\mathrm{S} / \mathrm{N})$ was set as first priority.

The observations were performed at the Gerostathopoulion Observatory of the University of Athens from July 2009 to December 2010 using various instrumentation setups. In particular, we used a $40-\mathrm{cm}$ Cassegrain telescope $\left(T_{1}\right)$, a 25-cm $\left(T_{2}\right)$ and a $20-\mathrm{cm}\left(T_{3}\right)$ Newtonian reflector telescope, and the ST10XME $\left(C_{1}\right)$ and ST-8XMEI $\left(C_{2}\right)$ CCDs equipped with the Bessell B, V, R, I photometric filters.

Differential magnitudes with the method of aperture photometry were obtained using the software MuniWin v.1.1.26 (Hroch 1998). In Table 1 we list: The number of nights $(N)$ of observations in a given time range $(T)$, namely the time difference in days between the last and first observation nights, the total time span (T.S.), the standard deviation (S.D.) of the observations (mean value), the abbreviation of the instrumentation (Ins) and the comparison $(C)$ and check $(K)$ stars used for each case. The spectral types of the stars were estimated according to their B-V indexes by using the tables published by Cox (2000), while the absolute magnitude $M_{v}$ was calculated from the relation given by McNamara \& Feltz (1978):

$$
M_{v}=-3.25(12) * \log P-1.45(11)
$$

where $P$ is the fundamental pulsation period in days. In Table 2 the apparent magnitudes in $\mathrm{B}$ and $\mathrm{V}$ passbands as given in The Tycho-2 Catalogue (Hog et al. 2000), the calculated B-V index, the spectral type range and the absolute magnitude of each star are presented.

Table 1: The observations log

\begin{tabular}{|c|c|c|c|c|c|c|}
\hline Star & $\begin{array}{c}\mathbf{N} \\
\text { [nights] }\end{array}$ & $\begin{array}{c}\mathbf{T} \\
\text { [days] }\end{array}$ & $\begin{array}{l}\text { T.S. } \\
{[\mathrm{hrs}]}\end{array}$ & $\begin{array}{c}\text { S.D. } \\
\text { [mmag] }\end{array}$ & Ins & $\begin{array}{l}\text { Comparison } \\
\text { Stars }\end{array}$ \\
\hline TYC 1134-414-1 & 5 & 12 & 22.4 & 5.1 & $T_{1}+C_{1}$ & $\begin{array}{l}\text { C: TYC 1134-247-1 } \\
\text { K: TYC 1134-735-1 }\end{array}$ \\
\hline TYC 1270-926-1 & 4 & 28 & 27.7 & 7.1 & $T_{2}+C_{2}$ & $\begin{array}{l}\text { C: TYC } 1274-1358-1 \\
\text { K: TYC } 1274-1387-1\end{array}$ \\
\hline TYC 1626-1303-1 & 7 & 9 & 26.7 & 5.1 & $T_{1}+C_{1}$ & $\begin{array}{l}\text { C: TYC } 1626-1290-1 \\
\text { K: TYC } 1626-1275-1\end{array}$ \\
\hline TYC 3164-1517-1 & 23 & 54 & 130.1 & 5.7 & $T_{1}+C_{1}$ & $\begin{array}{l}\text { C: TYC 3164-0083-1 } \\
\text { K: TYC 3164-0269-1 }\end{array}$ \\
\hline TYC 4559-2536-1 & 9 & 21 & 40.4 & 7.9 & $T_{3}+C_{2}$ & $\begin{array}{l}\text { C: TYC 4559-0189-1 } \\
\text { K: SAO } 8107\end{array}$ \\
\hline
\end{tabular}


Table 2: Magnitude information and spectral type estimations of the stars

\begin{tabular}{lccccc}
\hline Star & B & V & B-V & Sp. Type & $\mathbf{M}_{\boldsymbol{v}}$ \\
\hline TYC 1134-414-1 & $9.51(2)$ & $9.24(2)$ & $0.27(3)$ & A8-F0 & $2.9(5)$ \\
TYC 1270-926-1 & $12.3(2)$ & $11.5(2)$ & $0.8(3)$ & F7-K4 & $2.0(5)$ \\
TYC 1626-1303-1 & $12.2(2)$ & $12.3(2)$ & $-0.1(3)$ & O2-A7 & $3.5(5)$ \\
TYC 3164-1517-1 & $10.85(5)$ & $10.53(6)$ & $0.32(8)$ & A8-F4 & $1.5(5)$ \\
TYC 4559-2536-1 & $12.6(2)$ & $12.3(2)$ & $0.3(3)$ & A1-G1 & $2.5(5)$ \\
\hline
\end{tabular}

\section{Results of frequency analyses}

Frequency-analysis of all available data in the interval from 0 to $80 \mathrm{c} / \mathrm{d}$ was performed. The software PERIOD04 v.1.2, which is based on the classical Fourier analysis (Lenz \& Breger 2005), was used. The typical frequency range for $\delta$ Scuti stars is considered to be between 3-80 c/d (Breger 2000), while frequencies between $0-3 \mathrm{c} / \mathrm{d}$ could be caused potentially by atmospheric reasons or observational drifts. After the first frequency computation, the residuals were subsequently pre-whitened for the next one. The results of the frequency search for all cases are presented in Table 3 , where the identification number of the frequency $(N o)$, the frequency value $(F)$, its corresponding amplitude $(A)$ and phase $(\Phi)$ and the signal-to-noise ratio $(S / N)$ after pre-whitening for the previous frequency(ies) are listed. The error for each value, given in parenthesis, was calculated from the programme, which uses the least squares method. In addition, the sum of the squared residuals $\left(\chi^{2}\right)$ derived from a multi-parameter least-squares fit of sinusoidal functions, is also given for each adopted solution. The frequency spectra, the Fourier fits on the observational points for the longest (data) sets of observations and the spectral window of each star are illustrated in Figs. 1-4. 
Table 3: The frequency analysis results

\begin{tabular}{|c|c|c|c|c|c|c|c|c|}
\hline & \multicolumn{4}{|c|}{ TYC 1270-926-1 } & \multicolumn{4}{|c|}{ TYC 1626-1303-1 } \\
\hline No & $\begin{array}{c}\mathbf{F} \\
{[\mathrm{c} / \mathrm{d}]}\end{array}$ & $\begin{array}{c}\mathbf{A} \\
{[\mathrm{mmag}]}\end{array}$ & $\begin{array}{c}\phi \\
{[\mathrm{deg}]}\end{array}$ & $\mathrm{S} / \mathrm{N}$ & $\begin{array}{c}\mathbf{F} \\
{[\mathrm{c} / \mathrm{d}]}\end{array}$ & $\begin{array}{c}\mathbf{A} \\
{[\mathrm{mmag}]}\end{array}$ & $\begin{array}{c}\phi \\
{[\mathrm{deg}]}\end{array}$ & $\mathrm{S} / \mathrm{N}$ \\
\hline$f_{1}$ & $11.437(1)$ & $18.1(7)$ & $34(2)$ & 19.2 & $32.682(7)$ & $3.6(3)$ & $279(5)$ & 10.1 \\
\hline$f_{2}$ & $0.366(1)$ & $15.8(7)$ & $280(3)$ & 47.5 & $39.751(9)$ & $2.6(3)$ & $223(7)$ & 8.7 \\
\hline$f_{3}$ & $10.643(1)$ & $13.0(7)$ & $15(3)$ & 16.1 & $33.985(7)$ & $3.3(3)$ & $355(5)$ & 9.2 \\
\hline$f_{4}$ & $15.334(1)$ & $12.1(7)$ & $306(3)$ & 14.4 & $32.869(7)$ & $3.3(3)$ & $78(5)$ & 9.7 \\
\hline$f_{5}$ & $21.050(2)$ & $5.9(7)$ & $63(7)$ & 6.8 & $37.653(10)$ & $2.5(3)$ & $157(7)$ & 8.1 \\
\hline$f_{6}$ & $17.063(2)$ & $6.9(7)$ & $16(6)$ & 9.6 & $68.636(14)$ & $1.7(3)$ & $54(11)$ & 4.4 \\
\hline$f_{7}$ & $2.339(1)$ & $8.0(7)$ & $286(5)$ & 9.8 & $24.270(14)$ & $1.7(3)$ & $218(11)$ & 4.1 \\
\hline$f_{8}$ & $24.548(3)$ & $4.1(7)$ & $9(10)$ & 7.2 & $42.633(16)$ & $1.5(3)$ & $280(12)$ & 4.4 \\
\hline$f_{9}$ & 72.964 (3) & $3.4(7)$ & $186(12)$ & 4.0 & - & - & - & - \\
\hline$f_{10}$ & $5.653(2)$ & $5.2(7)$ & $98(8)$ & 6.7 & - & - & - & - \\
\hline$\chi^{2}$ & \multicolumn{4}{|c|}{0.002} & \multicolumn{4}{|c|}{0.005} \\
\hline & \multicolumn{4}{|c|}{ TYC 1134-414-1 } & \multicolumn{4}{|c|}{ TYC 4559-2536-1 } \\
\hline No & $\begin{array}{c}\mathbf{F} \\
{[\mathrm{c} / \mathrm{d}]}\end{array}$ & $\begin{array}{c}\mathbf{A} \\
{[\mathrm{mmag}]}\end{array}$ & $\begin{array}{c}\phi \\
{[\mathrm{deg}]}\end{array}$ & $\mathrm{S} / \mathrm{N}$ & $\begin{array}{c}\mathbf{F} \\
{[\mathrm{c} / \mathrm{d}]}\end{array}$ & $\begin{array}{c}\mathbf{A} \\
{[\mathrm{mmag}]}\end{array}$ & $\begin{array}{c}\Phi \\
{[\mathrm{deg}]}\end{array}$ & $\mathrm{S} / \mathrm{N}$ \\
\hline$f_{1}$ & $21.365(2)$ & $3.0(1)$ & $201(3)$ & 24.5 & $16.516(1)$ & $16.9(5)$ & $347(2)$ & 29.0 \\
\hline$f_{2}$ & $21.775(3)$ & $2.3(1)$ & $189(3)$ & 19.7 & $17.156(1)$ & $21.4(5)$ & $164(1)$ & 41.1 \\
\hline$f_{3}$ & $7.736(6)$ & $1.1(1)$ & $337(7)$ & 5.2 & $17.042(1)$ & $15.2(5)$ & $37(2)$ & 28.7 \\
\hline$f_{4}$ & $19.237(6)$ & $1.2(1)$ & $217(7)$ & 9.8 & $19.524(2)$ & $6.3(5)$ & $181(4)$ & 9.4 \\
\hline$f_{5}$ & $14.852(8)$ & $0.8(1)$ & $257(9)$ & 3.0 & $23.186(3)$ & $4.2(5)$ & $308(7)$ & 5.7 \\
\hline$f_{6}$ & $26.699(9)$ & $0.8(1)$ & $215(10)$ & 4.5 & - & - & - & - \\
\hline$\chi^{2}$ & \multicolumn{4}{|c|}{0.003} & \multicolumn{4}{|c|}{0.010} \\
\hline & \multicolumn{4}{|c|}{ TYC 3164-1517-1 } & & & & \\
\hline No & $\begin{array}{c}\mathbf{F} \\
{[\mathrm{c} / \mathrm{d}]}\end{array}$ & $\begin{array}{c}\mathbf{A} \\
{[\mathrm{mmag}]}\end{array}$ & $\begin{array}{c}\phi \\
{[\mathrm{deg}]}\end{array}$ & $\mathrm{S} / \mathrm{N}$ & & & & \\
\hline$f_{1}$ & $8.226(3)$ & $8.9(2)$ & $120(1)$ & 12.8 & & & & \\
\hline$\chi^{2}$ & \multicolumn{4}{|c|}{0.010} & & & & \\
\hline
\end{tabular}




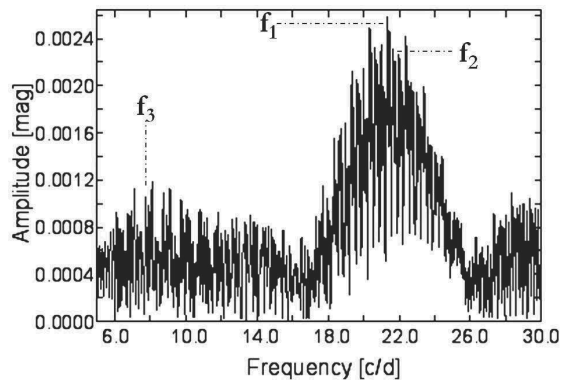

$\left(a_{1}\right)$

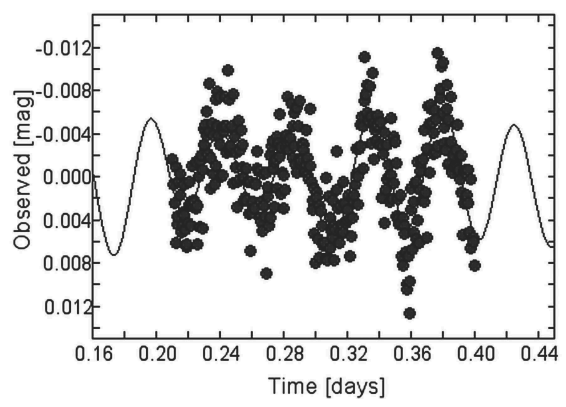

(b)

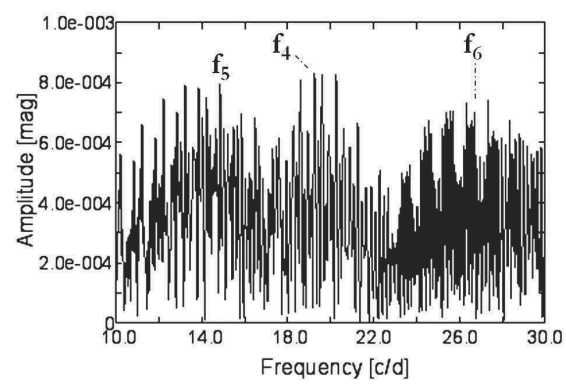

$\left(a_{2}\right)$

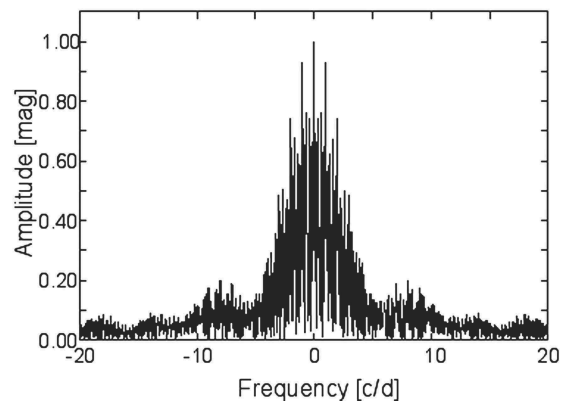

(c)

Figure 1: $\left(a_{1}\right),\left(a_{2}\right)$ : The Frequency spectra where the identified frequencies are indicated, (b): the Fourier fit on the observational points for the longest (data) set of observations, and (c): the spectral window for TYC 1134-414-1. 


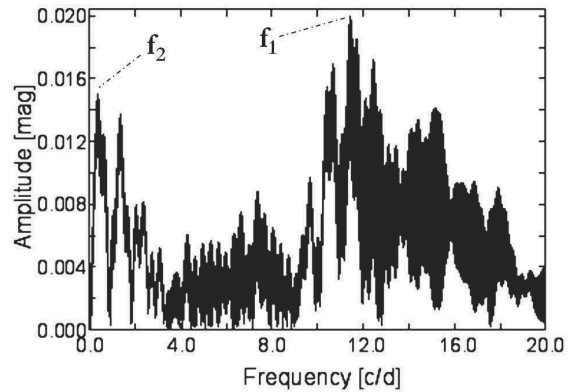

$\left(a_{1}\right)$

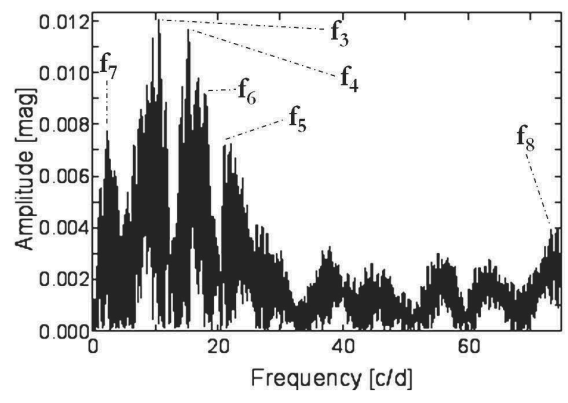

$\left(a_{2}\right)$

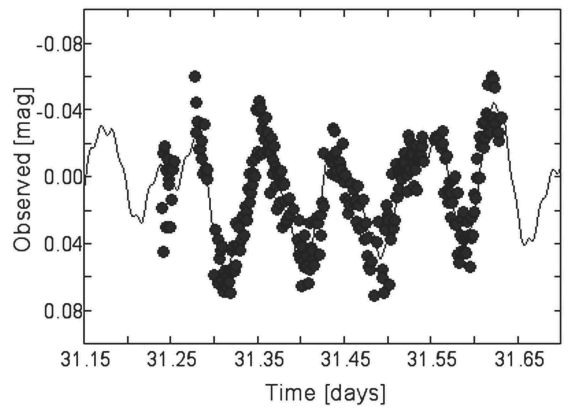

(b)

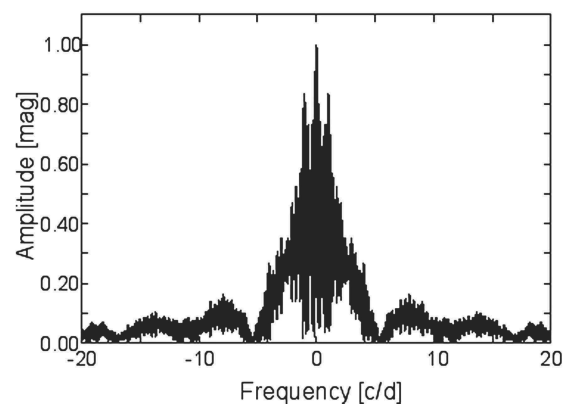

(c)

Figure 2: The same as Fig. 1 but for TYC 1270-926-1. 


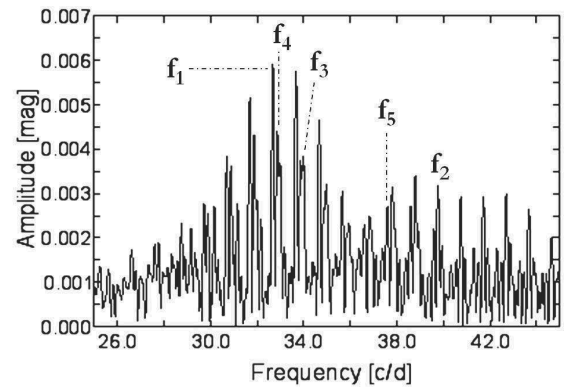

$\left(a_{1}\right)$

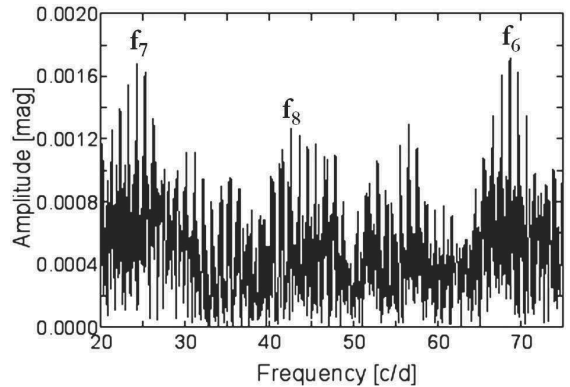

$\left(\mathrm{a}_{2}\right)$

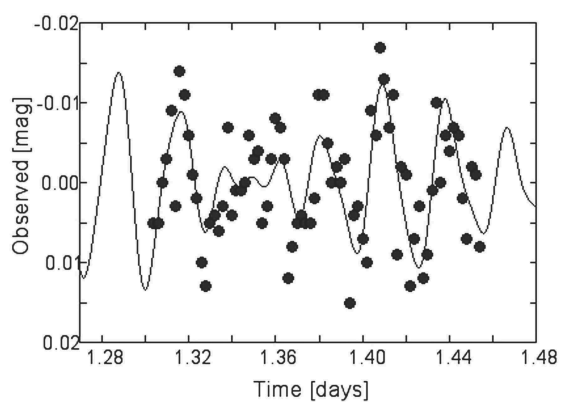

(b)

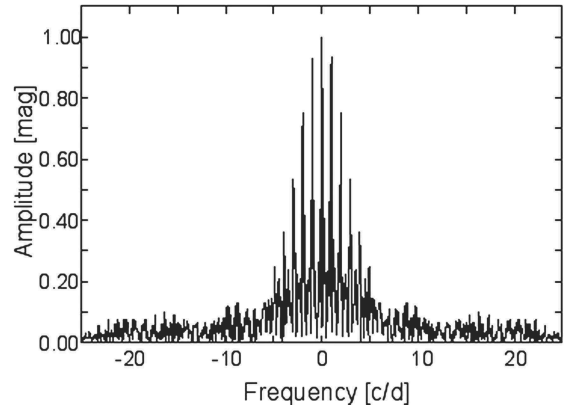

(c)

Figure 3: The same as Fig. 1 but for TYC 1626-1303-1. 


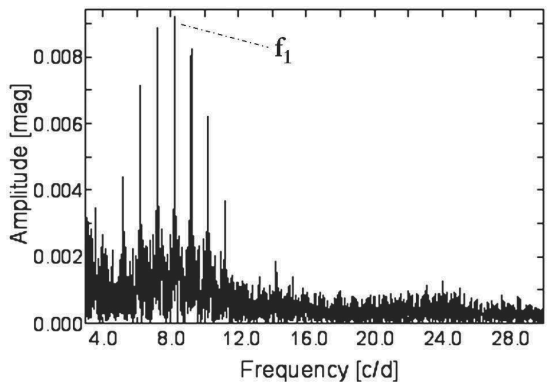

(a)

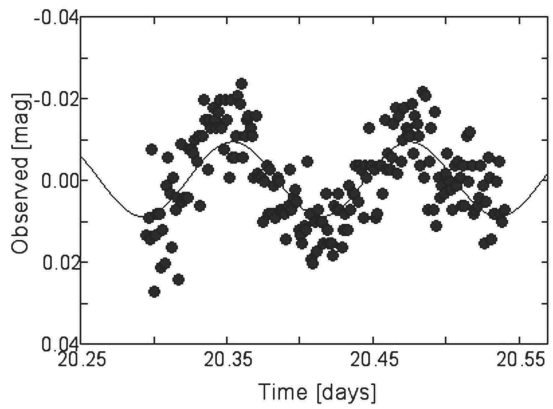

(c)

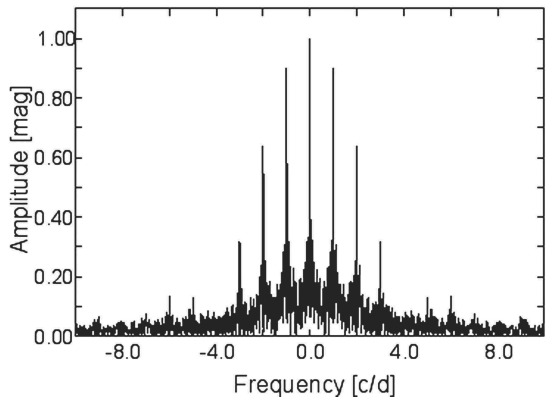

(e)

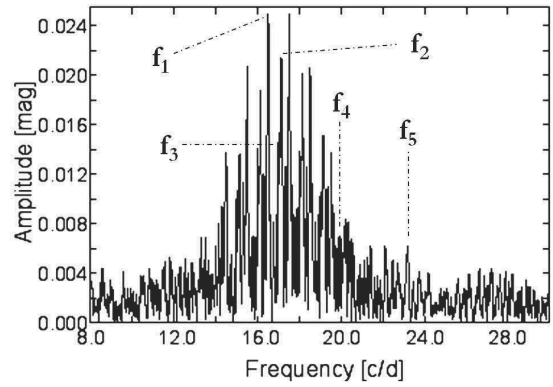

(b)

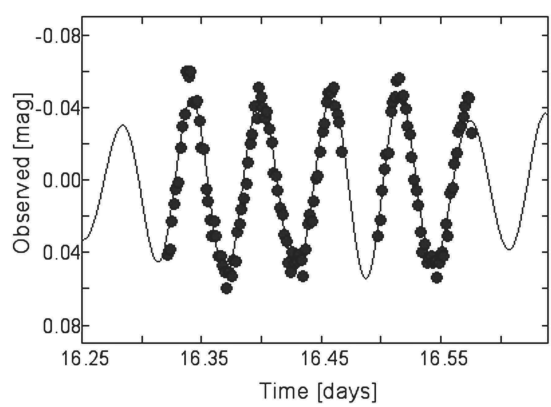

(d)

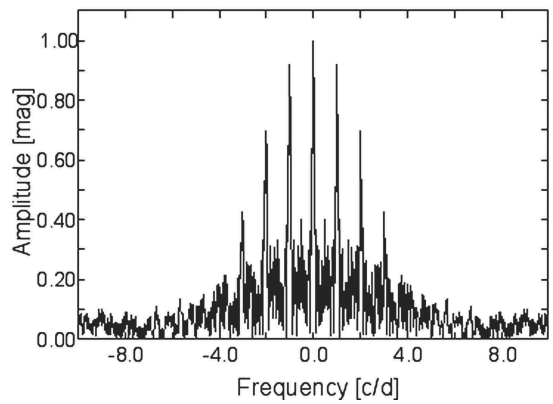

$(\mathrm{f})$

Figure 4: The same as Fig. 1, but (a), (c), (e) for TYC 3164-1517-1, and (b), (d), (f) for TYC 4559-2536-1. 
TYC 1134-414-1: Six possible pulsation frequencies were obtained for this star. $f_{1}$ and $f_{2}$ appear to have very close values, constituting very probably a close frequency pair, since the criterion of Loumos \& Deeming (1978) is well fulfilled. In particular, the half difference of $f_{1}$ and $f_{2}$ yields a value of $\sim 0.21$ $c / d$, which is larger than the achieved frequency resolution of $1.5 / T=0.125$ $\mathrm{c} / \mathrm{d}$, according to our time range (see Table 1 ).

TYC 1270-926-1: The frequency analysis revealed ten oscillating frequencies for this star. $f_{2}$ and $f_{7}$ are not inside the typical range for $\delta$ Scuti stars, therefore their origin could be non-physical (i.e. observational drifts, atmospheric conditions). Moreover, the ratio $f_{4} / f_{1} \sim 0.75$ is typical for the radial fundamental and first overtone modes.

TYC 1626-1303-1: In this case, eight frequencies were traced. The values of $f_{1}$ and $f_{4}$ differ by only $0.187 \mathrm{c} / \mathrm{d}$, and contrary to TYC $1134-414-1$, in this case a frequency resolution of $\sim 0.17 \mathrm{c} / \mathrm{d}$ was yielded. This value questions the real separation of these frequencies, since the criterion of Loumos \& Deeming (1978) is not satisfied. Due to this controversial issue, aliasing effects might implicate the results, hence another solution might be possible.

TYC 3164-1517-1: This 'slow' pulsator, unlike the other cases, was found to oscillate with a unique frequency of $8.226 \mathrm{c} / \mathrm{d}$.

TYC 4559-2536-1: The analysis for this star resulted in five frequencies. $f_{2}$ and $f_{3}$ yield a difference of $\sim 0.1 \mathrm{c} / \mathrm{d}$, they met the Loumos \& Deeming (1978) criterion, and therefore can be considered as a close frequency pair (Breger \& Bischof 2002).

\section{Discussion and conclusions}

In the present study five recently discovered pulsating stars are analysed with the Fourier method. Moreover, an approximate spectral classification is proposed. The combination of the above information provides the means for a coherent study of their intrinsic pulsational attributes and their classification as variable stars.

TYC 1134-414-1 was found to have six pulsation frequencies indicating a multi-periodic pulsator with radial and non-radial modes. Its spectral type, according to its $\mathrm{B}-\mathrm{V}$ index, is ranging between $\mathrm{A} 8-\mathrm{F} 0$, but a direct spectral observation of Turner et al. (2008) resulted into an A4/5 class. Our results are in agreement with those of Turner et al. (2008) as far as the dominant period is concerned but, in addition, more pulsation frequencies are presented in the current study, due to our larger time span. The star has already been classified as $\delta$ Scuti type.

TYC 1270-926-1 is a slight ambiguous case for classification. Although its 8-mode pulsational behaviour is certain, its spectral type was found to range 
between F7-K4. This value indicates a rather cool star, therefore it cannot be classified as a classical early-type pulsator.

As in the first case, TYC 1626-1303-1 is a multi-oscillating star, with eight frequencies identified. Its spectral type was found to be O2-A7, which partially covers both the range of $\delta$ Scuti and $\beta$ Cephei pulsating stars, but its absolute magnitude leads us to the conclusion that it could be a $\delta$ Scuti type pulsator (see Fig. 5).

TYC 3164-1517-1 has a spectral type between A8-F4 according to its B-V index. This result is consistent with its previously F0 classification (Heckmann 1975), placing it well inside the $\delta$ Scuti range. The frequency analysis revealed its mono-periodic oscillation.

TYC 4559-2536-1 can be classified as a $\delta$ Scuti pulsator with five frequencies in radial and non-radial modes, and a spectral type between A1-G1.

In Fig. 5 the position of each star with its uncertainties in the ColourMagnitude diagram is illustrated. The large errors come from the large uncertainty in their spectral types, derived from the B-V values.

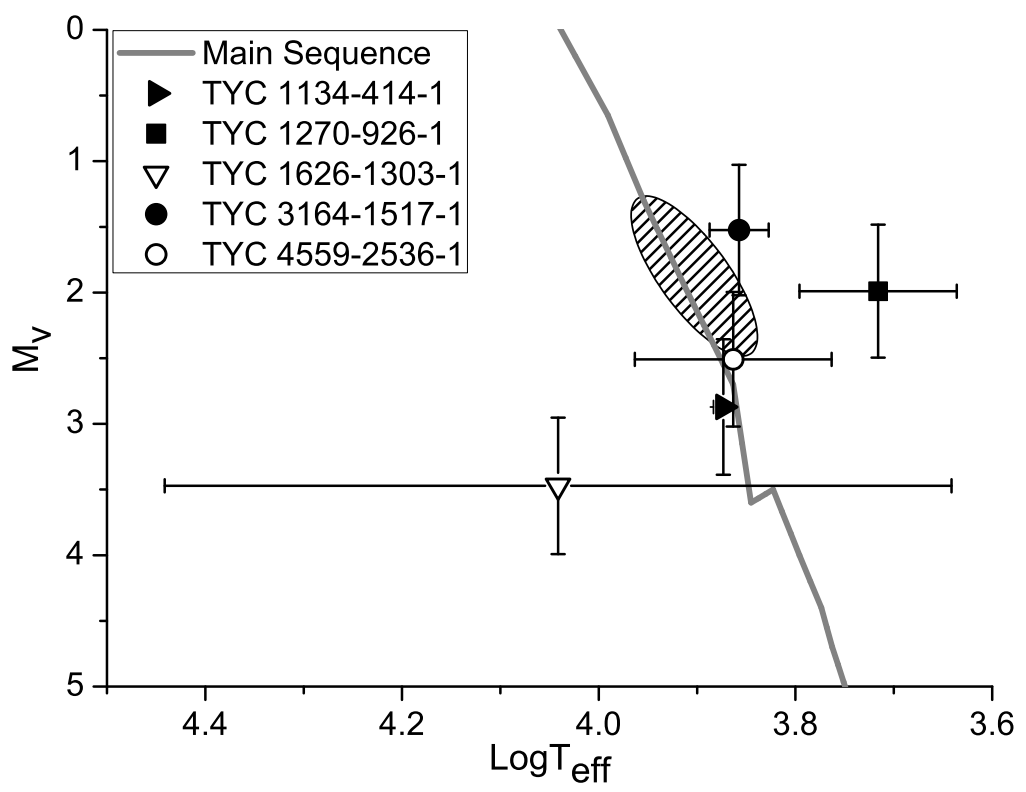

Figure 5: The location of the stars (symbols) in the Colour-Magnitude diagram. The dark grey solid line represents the Main Sequence, while the shaded ellipse corresponds to the $\delta$ Scuti region. 
For all stars, except TYC 1134-414-1 and TYC 3164-1517-1, spectroscopic observations will certainly improve the present classification of the stars, while photometric observations using larger telescopes in a larger time span will probably reveal more frequencies, which are below the detection threshold of the current instrumentation used for each case. In addition, for the case of TYC 1626-1303-1, where an ambiguous close pair of frequencies was found, photometric observations covering a larger time range will prove or disprove their separation.

Acknowledgments. This work has been financially supported by the Special Account for Research Grants No 70/4/9709 of the National \& Kapodistrian University of Athens, Hellas. The authors would like to thank the anonymous referee for valuable comments that improved significantly the present work.

\section{References}

Breger, M. 2000, ASPC, 210, 3

Breger, M. \& Bischof, K.M. 2002, A\&A, 385, 537

Cox, A.N. 2000, Allen's Astrophysical Quantities $4^{\text {th }}$ ed., Springer-AIP press

Heckmann O. 1975, AGK 3. Star catalogue of positions and proper motions north of -2.5 deg. declination, Hamburg-Bergedorf: Hamburger Sternwarte, 1975, edited by Dieckvoss, W.

Hog, E., Fabricius, C., Makarov, V.V., et al. 2000, A\&A, 355, 27

Hroch, F. 1998, Proceedings of the 29th Conference on Variable Star Research, 30

Liakos, A. \& Niarchos, P. 2011, IBVS, 6000, No 1, 2, 6 and 10, in press

Lenz, P. \& Breger, M. 2005, CoAst, 146, 53

Loumos, G.L. \& Deeming, T.J. 1978, ApSS, 56, 285

McNamara, D.H. \& Feltz, K.A., Jr. 1978, PASP, 90, 275

Turner, G., Kaitchuck, R., \& Skiff, B. 2008, JAVSO, 36, 156 